\title{
Effect of Excitation Parameters on the Barkhausen-Noise in FINEMET-Type Amorphous Ribbons
}

\author{
L. Daróczi ${ }^{1, *}$, G. Eszenyi ${ }^{1}$, Zs. Molnár ${ }^{1}$, D. L. Beke ${ }^{1}$, \\ A. Bükki-Deme ${ }^{1}$ and F. Zámborszky ${ }^{2}$ \\ ${ }^{1}$ Department of Solid State Physics, University of Debrecen, 4010 Debrecen, P.O. Box 2., Hungary \\ ${ }^{2}$ Magnetec Ungarn GmbH, H-3200, Gyöngyös, Pipis hegy, Hungary
}

\begin{abstract}
Probability frequency spectra of the peak area, energy and width of the Barkhausen noise (BN) signals have been measured versus induction in a narrow induction window during traversing the magnetization loop from negative to positive saturation (and reverse) in $\mathrm{Fe}(75) \mathrm{Si}(15) \mathrm{Nb}(3) \mathrm{B}(6) \mathrm{Cu}(1)$ amorphous metallic glasses. It was obtained, that the noise power, as the function of the induction, $B$, showed a wide minimum at $B=0$ and two sharp peaks around the knees of the magnetization loop. This can be a quite general feature of soft magnetic materials and can be related to the fact that at around the knees a mechanism, different from the domain motion in a random pinning field operates: new domains are created or existing domains are destroyed. Furthermore, it is also shown that the probability frequency of the duration, area and energy of the peaks are described by power function even far from the $B=0$ point, but with higher exponents belonging to the stationary values obtained around $B=0 . \quad$ [doi:10.2320/matertrans.M2013458]
\end{abstract}

(Received January 6, 2014; Accepted May 7, 2014; Published July 25, 2014)

Keywords: metallic glass, Barkhausen-noise, statistical analysis, excitation parameters, noise power

\section{Introduction}

It is well known that, Barkhausen noise $(\mathrm{BN})$ signals have very rich structure and contain information on different aspects of the magnetization process. ${ }^{1-8)}$ For example traversing the half-loop of the magnetization curve from saturation to saturation different mechanisms can contribute to the noise. In a limited induction interval around the coercive field, $H_{\mathrm{c}}$, (i.e., at $B=0$ on the $B-H$ hysteresis curve, where $B$ and $H$ are the magnetic induction and magnetic field) the differential permeability, $\mu=\mathrm{d} B / \mathrm{d} H$, can be approximately constant and the noise statistics can be considered stationary. ${ }^{8)}$ Here the domain wall motion in random pining field is likely to be the dominant magnetization process and theoretical descriptions, predicting e.g., the critical exponents of different Barkhausen noise parameters (e.g., in the probability frequency spectra of the area and duration of the peaks), apply and are in good agreement with experimental results. ${ }^{2-6,8}$ In these measurements care should be taken to measure and collect noise data indeed in a relatively narrow $B$ (or $H$ ) interval, where the permeability is constant. On the other hand, if the above observation window was shifted from saturation to saturation quite different behaviour was observed around the knees of the magnetization curve. ${ }^{1)}$ Indeed in Ref. 1), traversing the half-loop, a constant average induction rate $\mathrm{d} B / \mathrm{d} t$ was applied in $\mathrm{Fe}(3 \% \mathrm{Si})$ strips. The constant value of the magnetization rate was required because the statistical behaviour of the noise depends on the flux $\operatorname{rate}^{7-9)}$ and only this way a direct comparison of the results obtained at different stages of the magnetization process becomes possible. It was obtained in Refs. 1) and 7) that the noise power, $\langle P\rangle$ (the integral of the power spectrum, $S(f)$ where $f$ is the frequency) showed a wide minimum around $B=0$ and two peaks around the knees of the magnetization loop. The shape of the $B$ dependence of the noise power $(P)$ was described by the

*Corresponding author, E-mail: lajos.daroczi@science.unideb.hu following semi-empirical relation, $P=a \mu+b|\mathrm{~d} \mu / \mathrm{d} B|$, where $a$ and $b$ are constants. The above maxima were interpreted in Refs. 1, 7) as manifestations of large scale instabilities in which new domains were created or existing domains were destroyed (global domain rearrangements over the whole sample volume).

In one of our previous investigations on FINEMETtype ribbons ${ }^{10)}$ we found a definite correlation between the mechanical sensitivity, $\delta$ (determined by measuring the relative change of the diameter, $\varepsilon$, of a coiled cylindrical sample under compression: $\delta=\left(\mu_{0}-\mu_{\mathrm{a}}\right) / \varepsilon \mu_{0}$, where $\mu_{0}$ and $\mu_{\mathrm{a}}$ are the initial and the apparent values of the permeability), and the noise level of the peak area, or the total dissipated energy, $E_{\mathrm{t}}$, in annealed products. The total dissipated energy per one cycle was determined from the integral of the peak energy distribution function, $P(E) ; E=\int u^{2} \mathrm{~d} t$, where $u(t)$ and $t$ are the apparent voltage and the time, respectively and the integral runs from the start to the end of the individual peak. It is worth noting that $E_{\mathrm{t}}$ corresponds to the so-called resulting power of Barkhausen noise, averaged upon successive loops, and is the integral of the spectral density, $S(f)$, of the noise. It was shown in Ref. 10) that minimal mechanical sensitivity corresponds to a low magnetic Barkhausen noise and this fact can be used for diagnostic applications in industrial preparation of FINEMET-type or other ferromagnetic materials. In Ref. 10) there was not carried out a detailed analysis of the known power law, ${ }^{7}$ describing the probability frequency, $P$, of a given noise parameter, $x$ :

$$
P(x)=C x^{-\gamma} \exp \left(-x / x_{0}\right)
$$

( $x$ can be the duration, area, height or energy of the peaks, $C$ is constant, $\gamma$ is the so called critical exponent, and $x_{0}$ is the cut-off value). However, it can be seen from Fig. 4 of Ref. 10) that the power exponents of the peak area showed dramatic deviations (as the function of the crystalline fraction) from the value obtained in the initial amorphous ribbons for which the applicability of eq. (1) and the 
experimental value of $\gamma$ were confirmed from a detailed theoretical model based on motion of domain walls subject to random pinning field. ${ }^{3,4}$ Furthermore, in Ref. 11) the spectral density of the noise, measured under a constant magnetization rate in a narrow window centered around zero magnetization (covering about $8 \%$ of the linear part of the exciting triangular signal) was investigated in the same set of FINEMET-type ribbons as in Ref. 11). It was obtained that the high and low frequency portions of the spectral density of the noise displayed a definite correlation with the mechanical sensitivity parameter. These findings were in line with those obtained in Ref. 12): the spectral density of the noise exhibited a defined dependence on the deformation in cold rolled or tensile strained strips of low carbon steel, indirectly also illustrating that the parameters in eq. (1) can be different depending on the pre-history and/or the mechanism of the magnetization.

The above examples (all of them are also directly related to non-destructive testing) illustrate that it is interesting to investigate the kinetic exponents and the total dissipated energy (the resulting noise power) as a function of the position of the centre of the observation point traversing along the half magnetization loop from saturation to saturation in FINEMET-type materials, too. These investigations can answer the following questions: (i) is the shape of the $B$ dependence of the noise power similar or not in different soft magnetic materials, and (ii) how the characteristic exponents, determined in a narrow induction window with approximately constant permeability, depend on $B$.

\section{Experimental Procedure}

The measurements were carried out on melt spun, FINEMET-type amorphous $\mathrm{Fe}_{75} \mathrm{Si}_{15} \mathrm{Nb}_{3} \mathrm{~B}_{6} \mathrm{Cu}_{1}$ metallic glass samples. The $20 \mu \mathrm{m}$ thick and $10 \mathrm{~mm}$ wide ribbons were wounded onto a $32 \mathrm{~mm}$ diameter plastic tube in 4 layers forming a toroid core. The excitation coil with 360 turns was wounded uniformly onto the sample. The toroid sample was placed into a Permalloy shielding box. The concentrated detector coil ( $3 \mathrm{~mm}$ wide with 200 turns) was connected to a low noise preamplifier. The noise was acquisited (see the details in Ref. 11)) and then the different exponents of the $\mathrm{BN}$ were evaluated from the recorded data.

The excitation coil was powered with a triangle waveform generator producing constant field strength rate i.e., $\mathrm{d} H / \mathrm{d} t=$ const. The generator was connected to the excitation coil through high frequency chokes preventing undesirable feedback in the excitation circuit. The constant field strength rate results in different induction rates at different parts of the magnetization curve due to the variation of the local differential permeability, $\mu ; \mathrm{d} B / \mathrm{d} t=\mu \mathrm{d} H / \mathrm{d} t$.

For ensuring the constant induction rate through the full magnetization curve a simple solution was chosen. As a first step the quasi-static hysteresis loop of the sample was determined on a ring shaped sample by fluxmetric method at $0.5 \mathrm{~Hz}$ frequency. The upward (from negative to positive saturation) and downward branches of the hysteresis loop was divided into equidistant parts on the induction axis (Fig. 1).

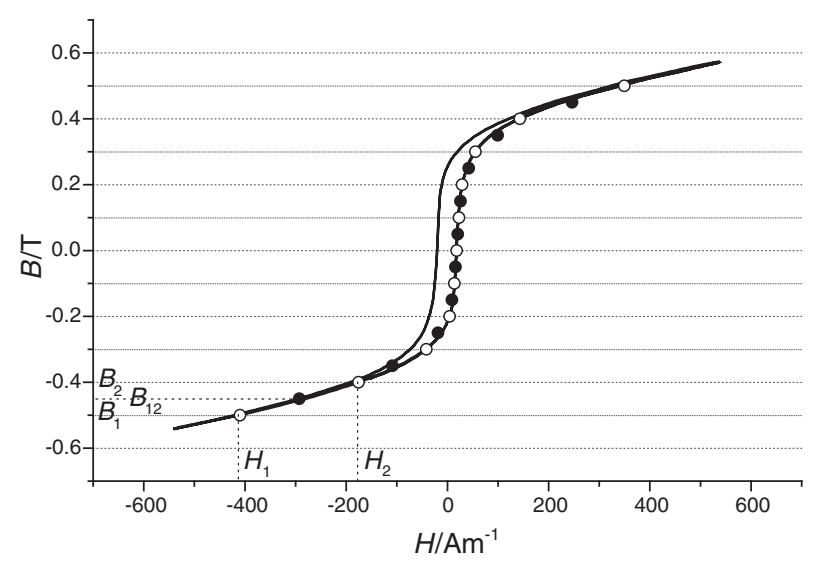

Fig. 1 Upward branch of the hysteresis loop with the borders (open circles) and centers (filled circles) of observation windows.

These equidistant induction regions (observation windows) have different average permeability values, determined by simple linear approximation within one window area (Fig. 1).

Using the average permeability values the field strength and excitation current rates can be determined for all observation windows, keeping a constant $(C)$ induction rate:

$$
\begin{aligned}
\mathrm{d} B / \mathrm{d} t & =C=\mu \mathrm{d} H / \mathrm{d} t=\mu k \mathrm{~d} I / \mathrm{d} t \\
\mathrm{~d} I / \mathrm{d} t & =C / \mu k=C^{\prime} / \mu
\end{aligned}
$$

where $I$ is the current of the generator, $k$ is geometrical constant.

Using the determined different current rates, corresponding to the constant $\mathrm{d} B / \mathrm{d} t$ induction rate (its value was about $50 \mathrm{mT} / \mathrm{s}$ ) about 20 full excitation cycles were performed and the Barkhausen signals were recorded only between the $H_{1}$ and $H_{2}$ field limits (e.g., for the $B_{1}-B_{2}$ window on Fig. 1). This process was repeated for all observation windows.

It is worth noting that since the demagnetization effect in the toroid geometry is negligible and the thickness of the toroid wall is small, one of the most important requirements for stationarity ${ }^{8)}$ is fulfilled in this arrangement.

Some preliminary relaxation heat treatments were carried out at low temperatures (below the crystallization temperature) for $1 \mathrm{~h}$ in order to relax the internal stresses, stored during the rapid solidification when the ribbons were produced. No systematic change in the characteristics of the noise was observed as the function of the relaxation temperature.

Additional measurements were carried out in solenoid configuration similar to the experimental arrangement in Refs. 2, 8). (Where the experimental conditions for stationary signal excitation and detection were summarized.) A sample with dimensions $0.02 \times 5 \times 300 \mathrm{~mm}$ was placed in a $600 \mathrm{~mm}$ long solenoid of $35 \mathrm{~mm}$ diameter. $4 \mathrm{~mm}$ long detector coil containing 400 turns was placed at the middle of the sample. The detector coil was connected to an amplifier of $1 \mathrm{~Hz}-$ $120 \mathrm{kHz}$ bandwidth. The amplified signal was recorded by a data acquisition unit (NI 6020E) with $200 \mathrm{ks} / \mathrm{s}$ sampling rate and the solenoid was driven by a voltage controlled current generator. External noises and undesirable feedbacks were reduced by the chokes and shielding. 
To improve the signal-to-noise ratio in this solenoid arrangement the sample was pre-stressed by a coil spring producing $\sigma=22 \mathrm{MPa}$ tensile stress. This is an important point for the comparison of the results of two sets of measurements: in the arrangement with toroid form no tensile stress was applied. It is known that the critical exponents determined in unstressed amorphous alloys are different (larger) from the one belonging to those obtained from stationary signals ${ }^{8)}$ measured in a stressed ribbon. The power exponents of the peak area, energy and duration distribution functions were determined under constant $(0.011 \mathrm{~T} / \mathrm{s})$ induction rate in a narrow (about $0.13 \mathrm{~T}$ width) observation window, located around the coercive field.

\section{Results and Discussions}

The following statistical properties of $\mathrm{BN}$ were determined: probability frequency of the peak duration (w), peak area (size),

$$
A=\int u(t) \mathrm{d} t
$$

and energy

$$
E=\int u^{2}(t) \mathrm{d} t
$$

where $u(t)$ is the detected Barkhausen-noise signal.

Nice fitting of the above probability distributions with eq. (1) was possible not only at around $B=0$ but for all $B$ values covering the full hysteresis loop. Figures 2 and 3 show, as an illustration, these functions for the peak area and peak energy at $B=0.175 \mathrm{~T}$ and $B=-0.255 \mathrm{~T}$, fields (on curve from negative to positive saturation) respectively. Thus from the probability distribution functions the values of the exponents were determined by numerical non-linear fitting using formula (1). The peak duration, size and energy exponents, denoted by $\alpha, \tau$ and $\varepsilon$ respectively, are plotted as a function of $B$ (Figs. 4, 5 and 6). The total dissipated energy per time length of the observation window (proportional to the average noise powers $\langle P\rangle$ ) at different $B$ values was determined by direct calculation from the recorded signal (Fig. 7).

Figures $8(a)-8(b)$ shows, as an illustration, the probability distributions functions for the peak size (a) and peak duration (b) at about $B=0$ as obtained from the measurements carried out in the second (solenoid) experimental set up. The values of the corresponding exponents are $\alpha=1.60 \pm 0.05, \tau=$ $1.45 \pm 0.05$ and $\varepsilon=1.37 \pm 0.05$, respectively.

The shapes of the curves shown in Fig. 7 suggest a similar interpretation used for the results of measurements in polycrystalline $\mathrm{Fe}(3 \% \mathrm{Si})$ strips. ${ }^{1)}$ In Ref. 1) it was obtained that the noise power showed a wide minimum around $B=0$ and two peaks around the knees of the magnetization loop. These maxima were interpreted in Refs. 1, 7) as manifestations of instabilities in which new domains were created or existing domains were destroyed, producing large scale instabilities (global domain rearrangements over the whole sample volume).

There is one more interesting feature of data shown in Fig. 7: the high asymmetry in the peak heights for the two
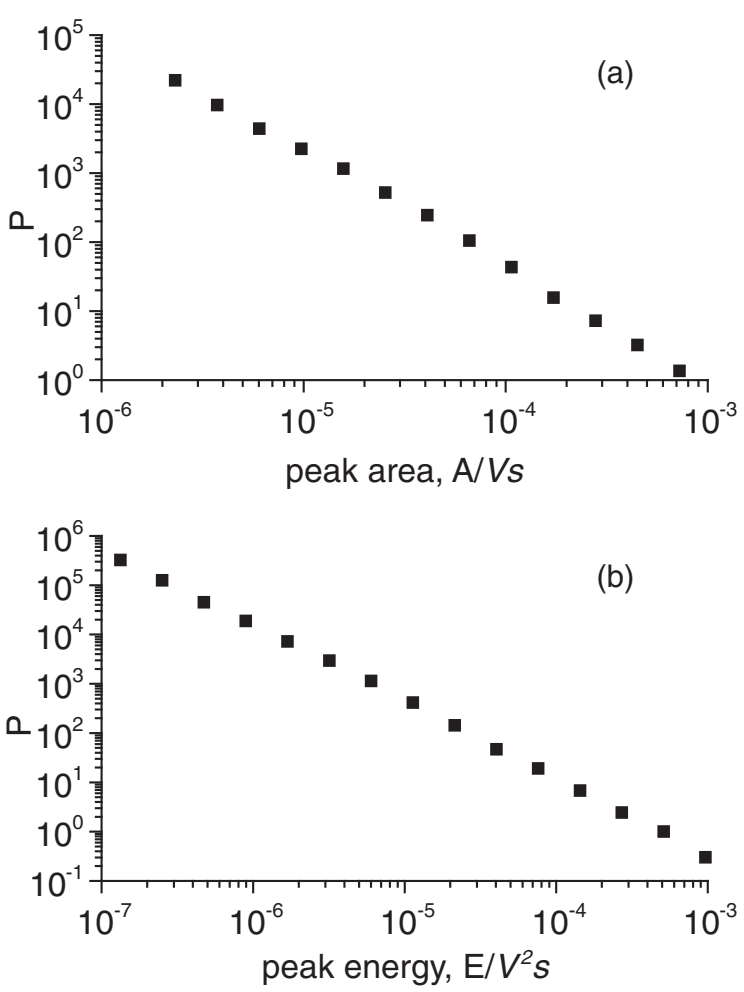

Fig. 2 Probability distribution functions for the peak size (a) and peak energy (b) at $B=0.175 \mathrm{~T}$ on curve from negative to positive saturation.
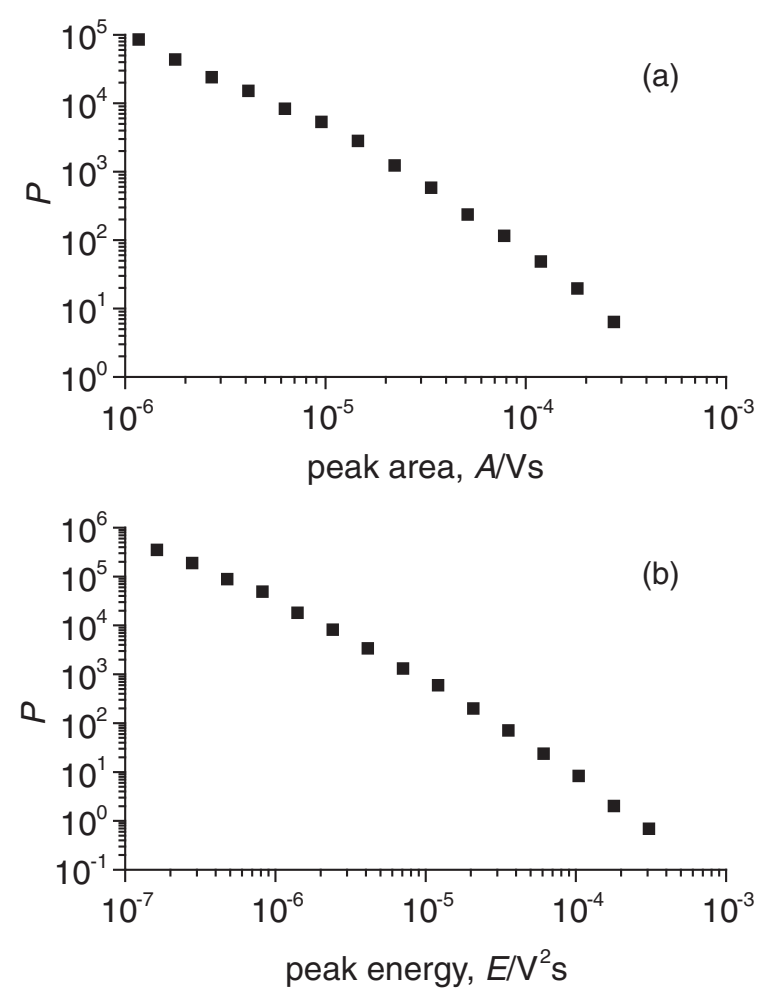

Fig. 3 Probability distribution functions for the peak size (a) and peak energy (b) at $B=-0.255 \mathrm{~T}$ on curve from negative to positive saturation.

different runs. Although it was not emphasized in Ref. 1) this asymmetry can also be seen in Fig. 3 of Ref. 1) (where only the run from negative to positive saturation was investigated) and is also suggested by the $P=a \mu+b|\mathrm{~d} \mu / \mathrm{d} B|$, empirical rule, since it also contains some asymmetry because the 

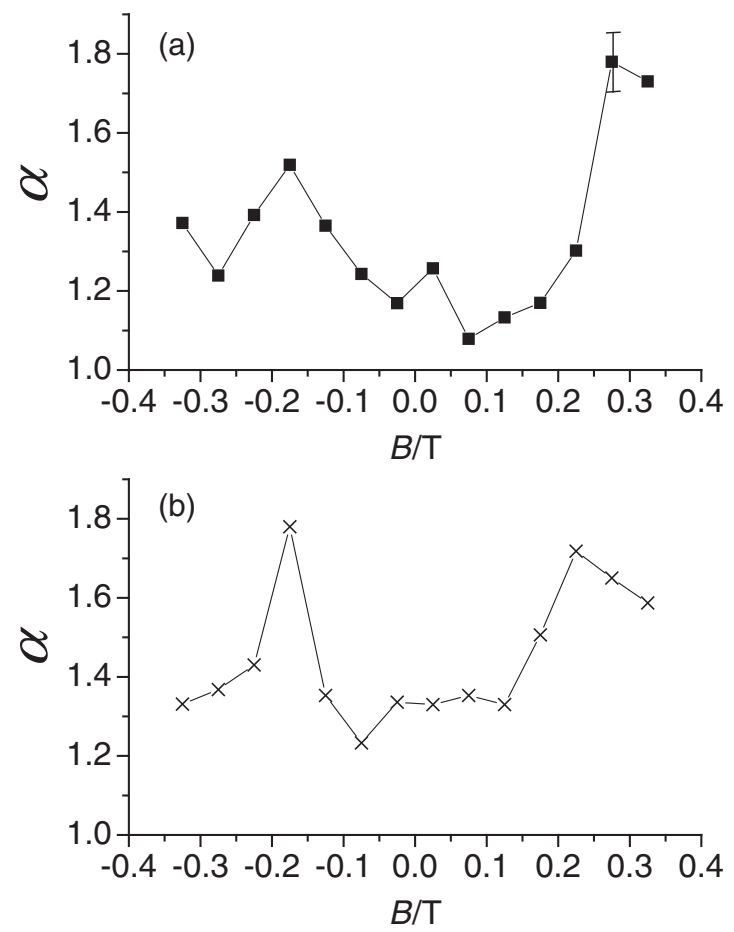

Fig. 4 Exponents $(\alpha)$ of probability distribution functions as the function of $B$ for the peak duration in runs from negative to positive (a) as well as from positive to negative saturation (b). A typical error of the date obtained is shown at one point.
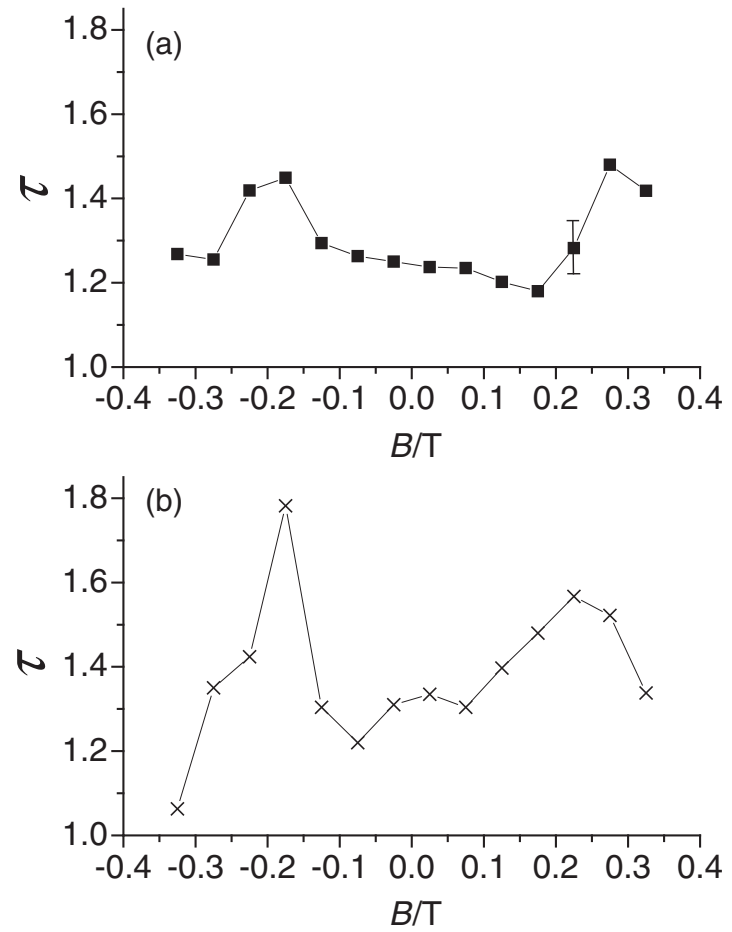

Fig. 5 Exponents $(\tau)$ of probability distribution functions as the function of $B$ for the peak size in runs from negative to positive (a) as well as from positive to negative saturation (b)

$\mathrm{d} \mu / \mathrm{d} B$ derivative has a bit different maximal values at negative and positive $B$, respectively. Our data shows that the total dissipated energy per time window (noise power) is always larger for the creation of new domains (at the knees belonging to the change of the magnetic structure from
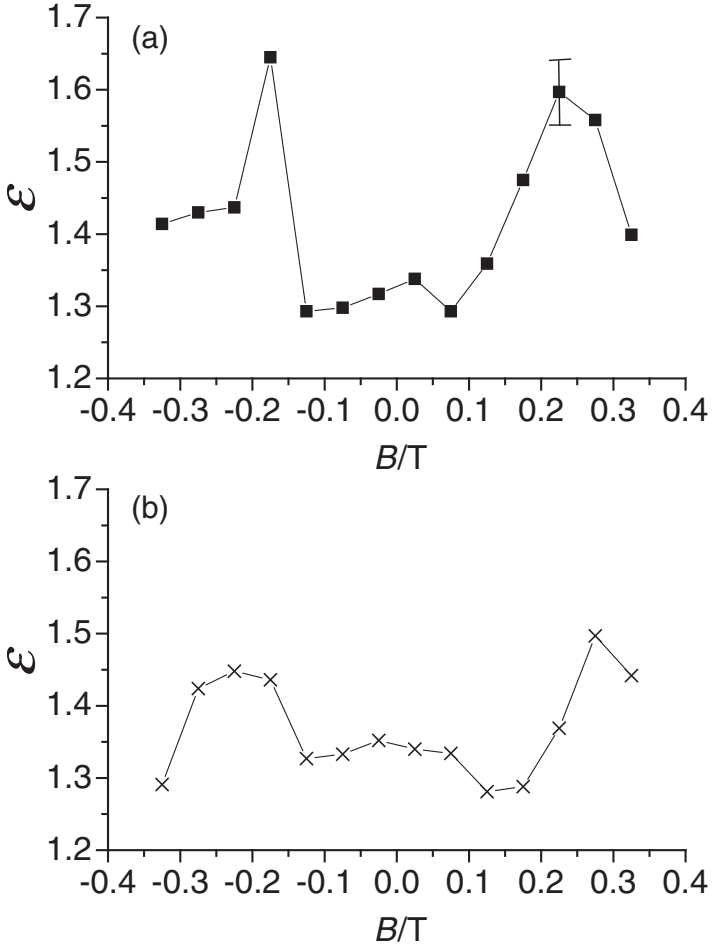

Fig. 6 Exponents $(\varepsilon)$ of probability distribution functions as the function of $B$ for the peak energy in runs from negative to positive (a) as well as from positive to negative saturation (b).
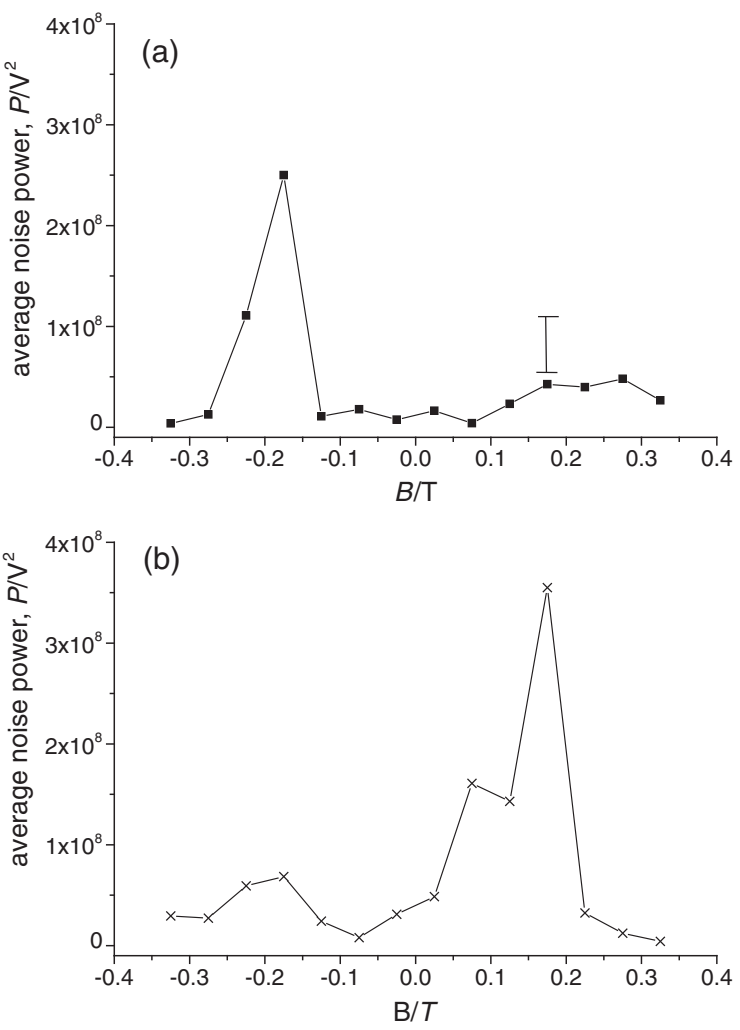

Fig. 7 Average noise power (i.e., total dissipated energy per time length of the observation window) versus the induction, $B$, in runs from negative to positive (a) as well as from positive to negative saturation (b).

saturation to multi domain structure) and has a much more moderate peak due to the disappearance of closely oriented domains (at the second knee on magnetization curve). 

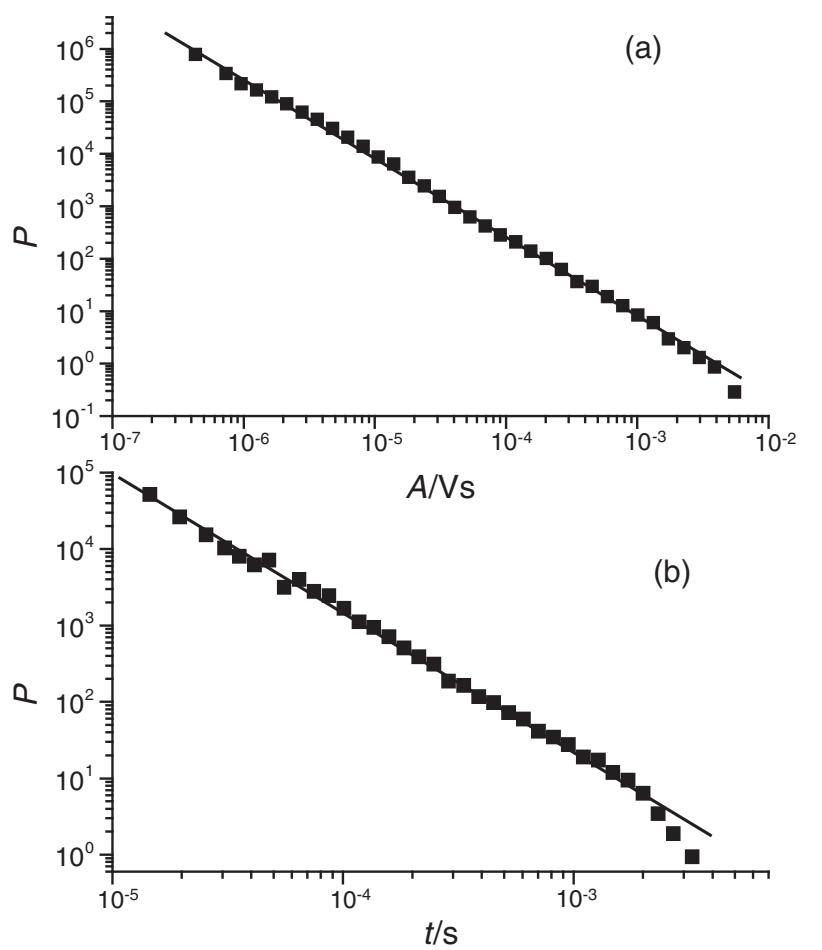

Fig. 8 Probability distribution curves obtained in the second (solenoid) arrangement shown for the peak area $A$ (a) and duration $T$ (b).

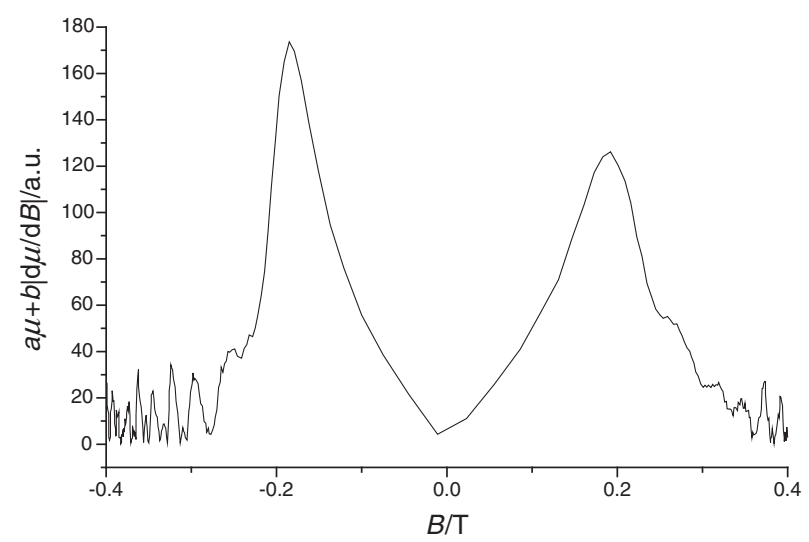

Fig. 9 The $P=a \mu+b|\mathrm{~d} \mu / \mathrm{d} B|$, values (as calculated from the data shown on Fig. 1) versus $B$ on the branch from negative to positive saturation.

Furthermore we can conclude that the critical exponents of the peak duration, size and energy show definite $B$ dependence (see Figs. 4, 5 and 6), reflecting the character of the $B$ dependence of the noise power.

In Ref. 1) the above semi-empirical relation was obtained from considerations based on a statistical model. Trying to fit it to our curves with appropriate fitting values of $a$ and $b$ it was obtained that this relation very well reproduced the positions of the maxima, but the difference in the heights of these maxima was much larger (the asymmetry was much more expressed) on the experimental curves, whatever the values of $a$ and $b$ were. As an illustration Fig. 9 shows this calculated function for the branch from negative to positive saturation for the fitting parameters $a=1$ and $b=1000$. Thus it can be concluded that the semi-empirical relation is not able to reflect fully the asymmetry of the peak heights observed.
The average values of the critical exponents $\alpha, \tau$ and $\varepsilon$ obtained in the arrangement shown in the second set up are in a good agreement with the values $\alpha=1.50 \pm 0.05 ;{ }^{3)}$ or $\alpha=1.65 \pm 0.08 ;^{8)} \tau=1.27 \pm 0.03,{ }^{5)}$ or $\tau=1.38 \pm 0.04 ;^{8)}$ considered belonging to stationary signals. Our values obtained at around $B=0$ in toroid arrangement are a little bit different from these: $\alpha \cong 1.35 \pm 0.10, \tau \equiv 1.30 \pm 0.10$ and $\varepsilon \equiv 1.35 \pm 0.5$. These deviations, although they are almost within the error bars, can be related to the fact that in the toroid arrangement no tensile stress was applied. The critical exponents can be different (larger) under uniaxial stress because the domain pattern is highly fragmented and dominated by residual stresses in quenched ribbons. ${ }^{8)}$ Nevertheless, we believe that the $B$ dependence of the noise power and the characteristic exponents obtained in the toroid arrangement reflects a general feature of soft magnetic materials. Furthermore, the $B$ dependence of the exponents is in line with the conclusion drown in Ref. 8): the critical exponents measured by sampling through the entire hysteresis loop (i.e., taking the average on $B$ in our figures) are higher than the values obtained from the narrow window around the coercive field.

It is worth mentioning that while in Ref. 8) the signals were collected by sampling the entire loop, and thus - because the permeability was not constant - the signals were non-stationary, in our case in each window the constant permeability condition was approximately fulfilled. Even in this case characteristic exponents have higher values far away from the window centered around the coercive field $(B=0)$.

\section{Conclusions}

From the noise power as well as the frequency spectra of the peak area, energy and width of the Barkhausen noise, measured versus induction in a narrow induction window during traversing the magnetization loop, not only from negative to positive saturation but in the reverse direction too, in $\mathrm{Fe}(75) \mathrm{Si}(15) \mathrm{Nb}(3) \mathrm{B}(6) \mathrm{Cu}(1)$ FINEMET-type amorphous metallic glasses the following results were obtained:

(1) The noise power, as the function of the induction, $B$, showed a wide minimum around $B=0$ and two peaks around the knees of the magnetization loop. This, together with the results of Ref. 1), illustrate that this is a quite general feature of (soft) magnetic materials.

(2) The noise power is always larger for the creation of new domains (at the knees belonging to the change of the magnetic structure from saturation to multi domain structure) and has a much more moderate peak due to disappearance of closely oriented domains (at the second knee, before the saturation on the magnetization curve).

(3) In relation to conclusion (2) the semi-empirical relation $P=a \mu+b|\mathrm{~d} \mu / \mathrm{d} B|$, is not able to predict fully the asymmetry of the peaks obtained.

(4) The probability frequency of the duration, area and energy of the peaks can also be described by a power function far from the $B=0$ point. The values of these exponents show a definite $B$ dependence reflecting the character of the $B$ dependence of the noise power. 


\section{Acknowledgments}

This work was carried out as part of the TÁMOP-4.2.2.A$11 / 1 /$ KONV-2012-0036 project in the framework of the New Hungarian Development Plan. The realization of this project is supported by the European Union, co-financed by the European Social Fund.

\section{REFERENCES}

1) G. Bertotti, F. Fiorillo and M. P. Sassi: J. Magn. Mag. Mater. 23 (1981) 136-148.

2) G. Bertotti and I. Mayergoyz: The Science of Hysteresis, 1st ed., Vol. 2, (Elsevier Inc., Amsterdam, The Netherlands, 2006) pp. 181-267.

3) G. Durin and S. Zapperi: Phys. Rev. Lett. 84 (2000) 4705-4708.

4) G. Durin and S. Zapperi: J. Appl. Phys. 85 (1999) 5196-5198.
5) L. Santi, F. Bohn, A. D. C. Viegas, G. Durin, A. Magni, R. Bonin, S. Zapperi and R. L. Sommer: Phys. B 384 (2006) 144-146.

6) M. Küpferling, F. Fiorillo, V. Basso, G. Bertotti and P. Meilland: J. Magn. Magn. Mater. 320 (2008) e527-e530.

7) G. Bertotti: Hysteresis in Magnetism, 1st ed., (Academic Press Inc., San Diego, 1998) pp. 281-296.

8) G. Durin and S. Zapperi: J. Stat. Mech. Theory and Experiment 1 (2006) P01002.

9) M. Celasco, F. Fiorillo and P. Mazzetti: Nuovo Cimento B 23 (1974) 376-384.

10) G. Eszenyi, S. Szabo, L. Harasztosi, F. Zamborszky, J. Nyeki, Z. Erdelyi and D. L. Beke: J. Mater. Res. 24 (2009) 130-134.

11) G. Eszenyi, A. Bükki-Deme, L. Harasztosi, F. Zámborszky, J. Nyéki, Z. Erdélyi, D. L. Beke and I. A. Szabó: J. Magn. Magn. Mater. 322 (2010) 322-325.

12) D. Spasojević, S. Bukvić, S. Milošević and E. H. Stanley: Phys. Rev. E 54 (1996) 2531-2546. 